\title{
EDITORIAL 7.1
}

Chères lectrices, chers lecteurs,

Ce matin je cherche sur yahoo.fr une illustration du tableau de René Magritte, «La trahison des images ». Par malheur, au lieu de taper le titre exact, j’écris les quelques mots, bien plus utilisés pour désigner l'œuvre, «Ceci n'est pas une pipe ». Mais Magritte n'est pas pour moi aujourd'hui. Voici qu'apparaît un message automatique : « Nous n'affichons aucun résultat pour votre recherche : celle-ci contient en effet un ou plusieurs mots susceptibles de fournir des résultats destinés à un public... . ». Machinalement, j'essaie les mêmes mots sur yahoo.com. Instantanément, l'image du tableau de Magritte est projetée sur mon écran - non, me dirait une de mes étudiantes du semestre qui s'achève, des «milliers d'images » de «Ceci n'est pas une pipe ».

Cette absurdité du système Internet ne me semble guère surprenante, mais je ne peux m'empêcher d'en faire part à mon collègue et rédacteur de Voix plurielles, Alexandre Amprimoz, à qui justement je téléphonais pour lui demander comment écrire un éditorial, exercice auquel je me prête pour la première fois. Alex répond d'ailleurs : «Tu écris ce que tu veux. »

Voilà, c'est fait. Bon, sur le principe, je crois fermement à un strict contrôle en certains domaines. Pourtant, en même temps, c'est vrai, je suis bien contente d'écrire pour une fois ce que je veux.

C'était d'ailleurs le critère de l'appel à articles pour ce numéro : « Sujets libres ».

J'espère que vous prendrez plaisir à lire ses articles, qui eux ont été strictement relus et contrôlés.

Catherine Parayre, Université Brock 